\title{
A Novel 8T SRAM Cell with Improved Read and Write Margins
}

\author{
Song li, Zhiting Lin*, Jiubai Zhang, Yuchun Peng and Xiulong Wu \\ School of Electronics and Information Engineering, AnHui University, Hefei, China \\ a11088@ahu.edu.cn
}

Keywords: Single-bit; SRAM; Stability; RSNM; HSNM; WM.

\begin{abstract}
A highly stable 8T SRAM cell is presented to improve the Static Noise Margin (SNM). The proposed 8T SRAM cell uses a single-bit line structure to perform read and write operation. The design enhances the write ability by breaking-up the feedback loop of the inverter pair. It also improves the read stability by eliminating the effects from the bit-line. The simulations show that the proposed $8 \mathrm{~T}$ cell offers $2.07 \mathrm{x}$ read static noise margin, $1.41 \mathrm{x}$ and $2.60 \mathrm{x}$ in write ' 0 ' margin compared to the conventional $6 \mathrm{~T}$ cell and $7 \mathrm{~T}$ cell, respectively. Besides, the proposed structure has a significant improvement in writing ' 1 ' operation and HSNM.
\end{abstract}

\section{Introduction}

According to ITRS road map, memory chips SRAM arrays will occupy most of the chip area in upcoming years. In order to achieve higher speed, performance and lower power consumption, the CMOS process technology is continuously scaling. Since the supply power has a significant impact on power consumption, voltage scaling is one of the most effective techniques for power reduction in digital VLSI design. However, it has some limitations like loss of static noise margin (SNM). And current fluctuations due to process variations and limitations on the number of cells connected to a single bit-line [1]. In the conventional 6T SRAM cell, the structure has a severe problem of read disturbing. One basic and effective method to eliminate this problem is the decoupling of true storing node from the bit lines during the read operation [2].

To improve the performance and data stability, several structures $[3,4,5]$ have been proposed at the cell level. For instance, a new 7T cell is proposed in [3], which improves the stability of the SRAM cell and reduces the average power dissipation during the read write operation and reduces the leakage power in standby mode. The usage of new devices such as FinFETs [6,7] has been suggested to improve the performance. In [6], the 8 SRAM cell structure was studied based on the $5 \mathrm{~nm}$ FinFET technology. This technique significantly increased the WM and has no adverse effect on the read SNM. Besides, the researchers have developed new methods $[8,9,10]$ to decrease power consumption.

A novel 8T SRAM cell is presented in this paper, using a single-bit line for read and write operations. The main contributions of the paper are listed as follows. First, the proposed cell improves both read and write noise margin as comparison with the conventional 6T SRAM cell. Second, the design only utilizes one bit line. Finally, compared with 6T SRAM cell, the proposed 8T SRAM cell has lower power consumption.

The rest of the paper is constructed as follows. The principal of the proposed 8T SRAM is reviewed in section two. In section three, the simulations results are presented and discussed. And section four concludes the paper.

\section{T SRAM Cell Design}

The schematic of the proposed 8T SRAM cell is presented in Fig.1 which uses single bit-line for read and write operations but has an extra world-line (RWL) for read. For a conventional 6T cell the worst-case Static Noise Margin (SNM) occurs in the read condition. This structural change is to improve the stability of the cell and eliminate the read disturb existing in the conventional 6T SRAM cell. In this SRAM cell design, transistor P3 is used to enhance the write margin and low power 
consumption of the circuit by breaking-up the feedback loop of the inverter pair. In next two subsections, we will introduce the operating principles of the proposed $8 \mathrm{~T}$ cell in detail.

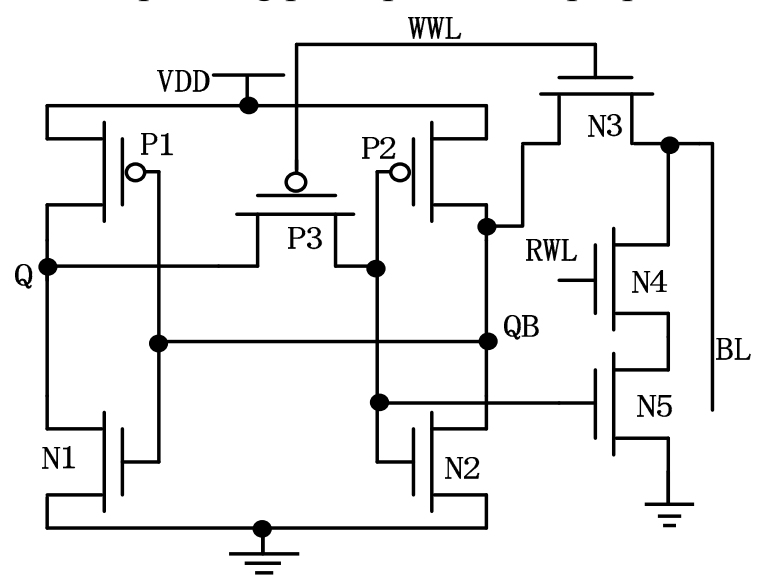

Fig. 1 The proposed 8T SRAM cell

Write Operation. A single-ended write design has comparative degradation in write performance in comparison to the conventional write structure using complementary bit-line pair. Nevertheless a dynamic mechanism of cutting feedback loop can effectively improve the write ability in the proposed 8T cell. In the write operation, WWL is set to VDD to prepare a path from internal node 'QB 'to BL and break the feedback loop. The RWL is set to VDD in the entire write operation.

During write ' 0 ' operation, WWL is attached to high, while BL and RWL are at ground which can switch transistor N4 and N5 off. If ' 1 ' is stored at node 'QB', the gate of the transistor N2 will be pull down the ground and the node 'QB' retains at very low voltages instead of $0 \mathrm{~V}$. Thus the transistor P1 turns on and it pulls up the voltage of storage of node ' $Q$ '. On the other hand, during writing ' 1 ' operation, both RWL and BL are at ground and the voltage of 'QB' is pull up using transistor $\mathrm{P} 2$, which can force $\mathrm{QB}$ to change the state from ' 0 ' to ' 1 '.

Read Operation. In our proposed 8T SRAM cell, the stored nodes are decoupled from the bit-line which eliminates the read disturb and presents better RSNM compared to the conventional 6T SRAM cell. For the read operation, the BL is pre-charged VDD initially. We can judge the stored data by depending on the state of BL. The proposed 8T SRAM cell improves the RSNM by at least 2.06x and as compared with conventional 6T SRAM cell. During the read operation, the bit-line is pre-charged to VDD, RWL is set to high. The WWL is set to low in the entire read operation, which turns off and turns on $\mathrm{N} 3$ and $\mathrm{P} 3$. As ' $\mathrm{Q}$ ' node stores ' 1 ' $\operatorname{logic}\left(\mathrm{Q}={ }^{\prime} 1\right.$ ', $\mathrm{Q}={ }^{\prime} 0$ '), the transistors $\mathrm{N} 2$, $\mathrm{N} 5$ and $\mathrm{P} 1$ are on and $\mathrm{N} 1$ as well as $\mathrm{P} 2$ is off. The BL discharges through N4 and N5 to the ground. Similarly, when ' $\mathrm{Q}$ ' stores '0' logic $(\mathrm{Q}=$ ' 0 ', $\mathrm{QB}=$ ' 1 '), the transistors $\mathrm{P} 2$ and $\mathrm{N} 1$ are on and $\mathrm{P} 1, \mathrm{~N} 2, \mathrm{~N} 5$ are off. Thus, there is no discharging path from $\mathrm{BL}$ to ground.

\section{Simulation Results and Comparison}

In this section, simulation results have been obtained among conventional $6 \mathrm{~T}, 7 \mathrm{~T}$ and the proposed 8T SRAM cell. The RSNM and WM will be discussed in the section. All simulation results are done at SMIC 65nm technology.

Read Static Noise Margin (RSNM). As shown in Fig. 2, the analysis of the RSNM is based on 2000 samples Monte Carlo simulations during the read operation. The butterfly curves of 6T, 7T and 8T SRAM cells are shown in (a), (b) and (c) of Fig. 2 respectively, which indicate the read ability. The statistic shows that the proposed cell presents 2.06x improvement in RSNM as compared to 6T SRAM cell. Obviously, the proposed $8 \mathrm{~T}$ cell is superior to the $6 \mathrm{~T}$ cell in read operation. 


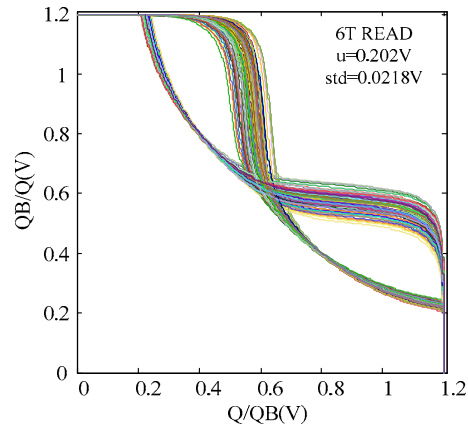

(a)

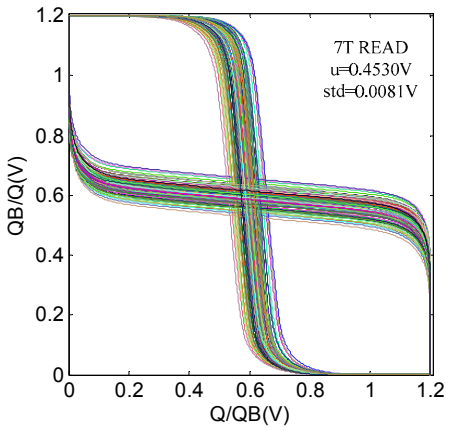

(b)

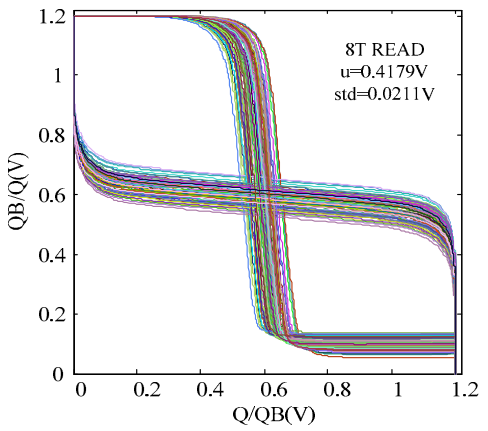

(c)

Fig.2 Read butterfly curves of SRAM cells

Hold Static Noise Margin (HSNM). Fig.3 shows the noise margin comparisons of SRAM cells during data retention. All statistical butterfly curves of SRAM cells have been carried out 3000 Monte Carlo simulations samples at low supply power. As shown in (a) and (b), the simulation results shows the $7 \mathrm{~T}$ is similar to $6 \mathrm{~T}$ cell in hold mod. But our proposed design (c) offers better hold stability and the lowest deviation as compared to 6T cell and 7T cell.

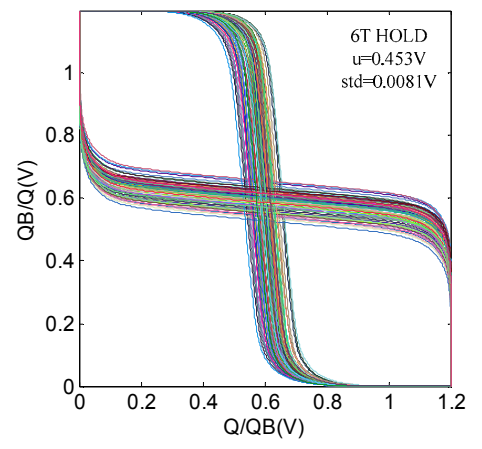

(a)

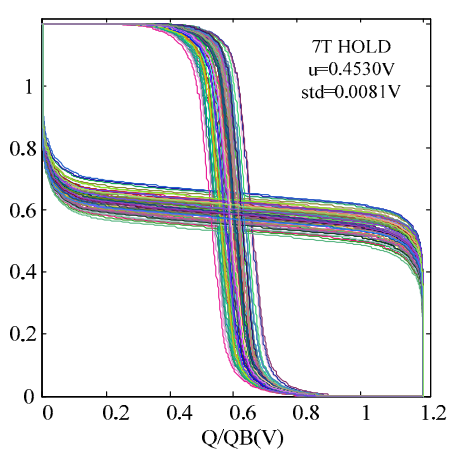

(b)

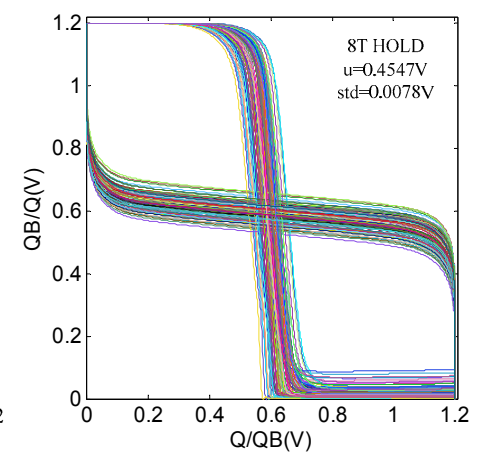

(c)

Fig.3 Hold butterfly curves of SRAM cells

Write Margin (WM). This new structure improves WM significantly, especially in writing ' 1 ' operation. As depicted in Fig. 4, the write ' 0 ' of our proposed design is enhanced (1.41x and 2.6x as compared to $6 \mathrm{~T}$ and $7 \mathrm{~T}$ ).

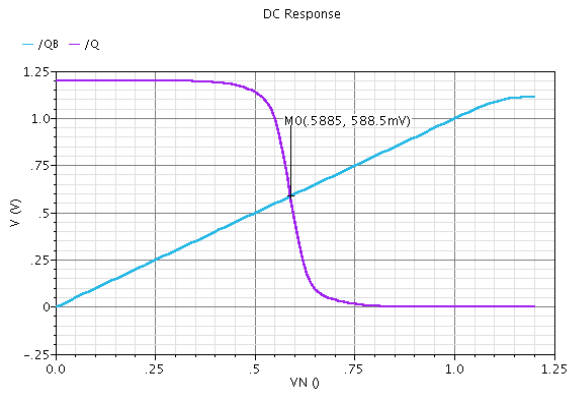

(a) $8 \mathrm{~T}$ Write ' 0 ' margin

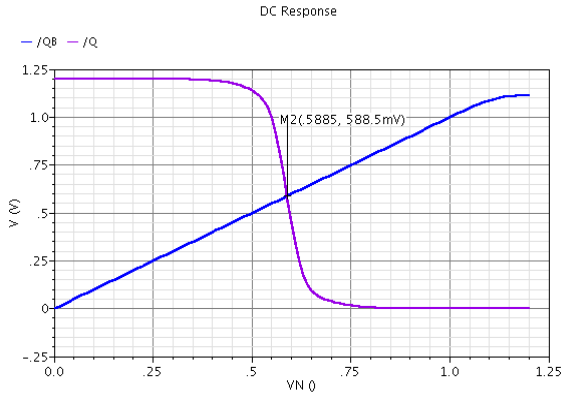

(b) $8 \mathrm{~T}$ Write ' 1 ' margin

Fig.4 WM of $6 \mathrm{~T}, 7 \mathrm{~T}$ and $8 \mathrm{~T}$

Power Consumption. TABLE I compares the read, write and hold power consumption of 6T, 7T and proposed 8T SRAM cells. The experimental results show that the average power consumption of the proposed design is lowest as compared to $6 \mathrm{~T}$ and $7 \mathrm{~T}$. 
Table I Power Consumption of Different Cells

\begin{tabular}{|c|c|c|c|c|c|}
\hline SRAM cell & $\operatorname{Read}^{\prime} 0 ’(\mathrm{~W})$ & Read'1'(uW) & Write`0’ (pW) & Write '1'(W & Hold $(\mathrm{pW})$ \\
\hline 6T SRAM & $52.69 \mathrm{u}$ & 52.69 & 103.8 & $103.8 \mathrm{p}$ & 139.8 \\
\hline 7T SRAM & $136 p$ & 52.68 & 143.6 & $52.69 \mathrm{u}$ & 179.6 \\
\hline 8T SRAM & $1.109 n$ & 52.68 & 104.8 & $598.1 p$ & 143.2 \\
\hline
\end{tabular}

\section{Summary}

A novel single ended 8T SRAM cell with high stability is proposed in this paper. The proposed cell has been found to perform better in read and write operations. While performing the write and read operation, the design enhances the WM and RSNM respectively. It breaks up the feedback loop of the inverter pair and obtains an excellent read robustness. Compared to the conventional 6T SRAM cell, the proposed cell exhibits $2.07 \mathrm{x}$ read static noise margin and $1.41 \mathrm{x}$ write margin. Besides, lower read and write power dissipation is obtained in this new structure.

\section{Acknowledgements}

This research is supported by natural science fund for colleges and universities in Anhui Province (KJ2013A006) and the 2014 Talents Project for Promoting Higher Education in Anhui Provincial.

\section{References}

[1] R. Gonzalez, B. Gordon, and M. Horowitz: Supply and threshold voltage scaling for low power CMOS, IEEE J. of Solid-State Circuits, Vol. 32, no.8, pp. 1210-1216, August, (1997)

[2] N. Verma and A. P. Chandrakasan: A $256 \mathrm{~kb} 65 \mathrm{~nm} 8 \mathrm{~T}$ subthshold SRAM employing sense-amplifer redundancy, IEEE J. Solid-State Circuits, vol. 43, no. 1, pp. 141-149, (2008)

[3] V. Kumarl, G. Khanna2: A Novel 7T SRAM cell Design for Reducing Leakage Power and Improved Stability, IEEE International Conference on Advanced Communication Control and Computing Technologies, pp. 56-59, (2014)

[4] C. B. Kushwah, S. K. Vishvakarma: A sub-threshold eight transistor (8T) SRAM cell design for stability improvement, IEEE International Conference on IC Design \& Technology (ICICDT), pp. $1-4,(2014)$

[5] C.Y. Lu, C.T. Chuang: A disturb-free subthreshold 9T SRAM cell with improved performance and variation tolerance, IEEE International SOC Conference (SOCC), pp. 325-329, (2013)

[6] R.Yarmand, B. Ebrahimi1, et al.: High-performance and High-Yield 5nm Underlapped FinFET SRAM Design using P-type Acess Transistor. IEEE 16th Intel Symposium on Quality Electronic Design, pp. 10-17, (2014)

[7] S.M. Salahuddin, H.L. Jiao, V. Kursun: Low-leakage hybrid FinFET SRAM cell with asymmetrical gate overlap / underlap bitline access transistors for enhanced read data stability". IEEE International Symposium on Circuits and Systems (ISCAS), pp. 2331-2334, (2013)

[8] R. Deena, K. Selvam, C. Senthilpari, L. Lini: Low power and low voltage SRAM design for LDPC codes hardware applications, IEEE International Conference on Semiconductor Electronics (ICSE), pp. 332-335, (2014)

[9] S. Arif, S. Pal: Variation-resilient CNFET-based 8T SRAM cell for ultra-low-power application. IEEE International Conference on Signal Processing And Communication Engineering Systems (SPACES), pp. 147 - 151, (2015)

[10] L. Wang, J.H. Wang, Z.Z. Yang, L.G. Hou, N. Gong: A Low Power CMOS Technology Compatible Non-volatile SRAM Cell. IEEE 12th International Conference on Solid-State and Integrated Circuit Technology (ICSICT), pp. 1-3, (2014) 